\title{
Growth of Discrete Projection Ghosts Created by Iteration
}

\author{
Imants Svalbe and Shekhar Chandra \\ School of Physics, Monash University, Australia
}

\begin{abstract}
Ghost images contain specially aligned pixels with intensities that are designed to sum to zero when projected at any of a pre-selected set of discrete angles. Ghost images find use in synthesizing the content of missing rows of image or projection space from data that contains some deliberate level of information redundancy. Here we examine the properties of ghost images that are constructed through a process of iterated convolution. An initial ghost is propagated by cumulative displacements into other discrete directions to expand the range of angles that have zero-sum projections. The discrete projection scheme used here is the finite Radon transform (FRT). We examine these accumulating ghosts to quantify the growth of their dynamic range of their pixel values and the spread of their spatial extent. After $N$ propagations, a pair of points with intensity \pm 1 can replicate to produce a maximum total intensity of $2^{N}$. For the discrete projections of the FRT, we show that columnoriented iterations better suppress the range and rate of growth of ghost image values. After $N$ row-based iterations, the peak pixel values of FRT ghost images grow approximately as $2^{0.8 N}$. After $N$ column-based iterations, the peak pixel values of FRT ghost images grow approximately as $2^{0.7 N}$. The slower rate of expansion of pixel values for column iteration comes at the expense of fragmenting the compactness of the set of FRT projection angles that are chosen to sum to zero.
\end{abstract}

\section{Introduction}

Ghosts are deceptively simple spatial images composed of signed pixel values, so arranged that projections of the ghost image data vanish or sum to zero for a range of pre-selected projection angles. The addition of linear combinations of scaled and shifted patterns of ghost values to a discrete object then adds nothing to the content of some of its projected views. Reconstruction of the object from its projected views is then either not unique or has an inconsistent interpretation, depending on the projected views that are taken.

Ghosts have formed an integral part in the post-Radon history of the reconstruction of digital images from discrete projections. Katz 9] and Louis [1] showed that the fidelity of reconstructed images is predicated on the banishment of ghosts or zero projections from the input data. Ghost theory gave rise to the Katz-criterion that specifies the number of acquired 1D projected views of an object that is sufficient for accurate reconstruction of a $2 \mathrm{D}$ slice. Images

I. Debled-Rennesson et al. (Eds.): DGCI 2011, LNCS 6607, pp. 406416, 2011.

(C) Springer-Verlag Berlin Heidelberg 2011 
are uniquely and exactly reconstructable from a set of projections if and only if the set of the projection angles constrains their ghost images to be supersets of the reconstructed image dimensions.

Gardner and Gritzman [5] and Barcucci et al [1] showed how polygonal arrangements of translated switching components could be used to generate ghosts at any selection of projection angles. Their work underlined the importance of these structures in fixing the sets that can be reconstructed uniquely from finite sets of projected sums. Zopf [17] examined ways to minimise the size of the arrangement of switching elements, a topic that is further addressed in Svalbe and Normand [14]. Herman and Davidi [8] examined the practical effect of adding ghosts to images reconstructed from sub-sampled sets of real projection data. The spatial extent and range of image intensities for ghosts that are created by iterated displacement of switching elements is the subject of this paper.

The discrete projection scheme that we use here is the finite Radon transform (FRT) developed by [12. These projections are applied to data embedded on prime-sized arrays. The FRT formalism relies on the unique and exact tiling in 2D space of oriented slices in discrete images and their associated discrete Fourier transforms. This "discrete slice theorem" approach was developed by [6].

Ghosts provide a bridge between the symmetric set of discrete projections of the FRT and the more general asymmetric sets of the discrete Mojette transform [7]. The distribution of pixel grey scales in a ghost and their spatial extent in image space are crucial aspects that govern how easily they can be implemented to de-convolve mixtures of ghosts. Reconstructions that use a projection set with one or more missing projections will generate artefacts in the reconstructed image. These image artefacts can be seen to be linear combinations of ghosts, as the projected image intensities must sum to zero for each missing angle. If the image contains regions that are known to be constant, then the added mixtures of ghosts will perturb these constant values. This property suggests a way to remove image reconstruction artefacts [4].

We examine and quantify the spatial spread and the rate at which the signed pixel values in a ghost image grow under a scheme where iterative convolutions are used to increment the size of the angle set for which the discrete projections will sum to zero. Ghosts created by this method become non-minimal after just a few iteration steps. Minimal ghosts span the full extent of the image space, but contain the theoretical minimum of $2 N$ pixels; $N$ with value +1 , and $N$ pixels with value -1 . Methods to generate minimal ghosts are presented in [15]. Examination of the properties of minimal ghosts is presented in a companion paper [14].

The finite Radon transform is reviewed in section 2 Section 3 outlines the method of constructing ghosts by iterative convolution. Section 4 gives some examples of ghosts constructed by iterations oriented along the row and column directions. Section 5 compares quantitatively the growth patterns of these two methods. Section [6 outlines some areas where this work may be extended and summarises our current findings. 


\section{Discrete Projections and the Finite Radon Transform}

The Finite Radon Transform (FRT) is a linear transform that preserves all of the information present in the original image. The image $I(x, y)$ is always exactly recoverable from its FRT $R(t, m)$. A fast algorithm to compute the FRT and its inverse, based on the number theoretic transform, was presented in [3]. A symmetrised form of the FRT enables a systematic redundant encoding of data makes it suitable for an error correcting internet transmission scheme [13. Ghosts form an inherent part of the data encoding method in the latter work.

We consider data displayed on a $p \times p$ image array, where $p$ is prime. The FRT projects this data at a set of $p+1$ discrete projection angles. Each projection is comprised of $p$ parallel translated rays. Each ray sums the intensity of exactly $p$ pixels located in the image domain. The FRT assumes that the data wraps periodically at the boundaries of the image. Then $I(x+m p, y+n p)=I(x, y)$ for any integers $m$ and $n$. The FRT, $R(t, m)$, of an image $I(x, y)$ is defined as

$$
R(t, m)=\sum I\left(\langle t+m y\rangle_{p}, y\right)
$$

where $0 \leqslant t<p, 0 \leqslant m \leqslant p$, and the notation $\langle j\rangle_{p}$ means taking the modulus (or integer remainder) of $j$ with respect to division by $p$. The sum $R(t, 0)$ is taken along column $t$ of the image, whilst the sum $R(t, p)$ is taken along the $t^{\text {th }}$ image row. A ghost at $N$ angles means $R(t, m)=0$ for all $t$ at $N$ values of $m$.

The discrete projection angle, $m$, is defined as the gradient of a line, or projection ray, linking pixels located at points $m$ steps across and one pixel down from each other. The projection angle $\tan ^{-1}(1 / m)$ can be re-mapped to conventional tomographic angles, with one unique angle for each value of $m$, as the direction of the ray that links nearest neighbour pixels that lie on a ray with the same value of $t$ [10. Then $\theta_{j}=\tan ^{-1}\left(y_{j} / x_{j}\right)$, where the vector linking nearest neighbour pixels has relative coordinates $\left(x_{j}, y_{j}\right)$. A scheme to perform exact discrete rotations of FRT projections was presented in [16]. Affine transformations, in the spatial domain or in FRT space, can be applied to perform discrete rotations on ghost images. The property of projections summing to zero for $N$ projection angles is retained under these transformations, provided that the affine mapping is invertible.

\section{Generating Ghosts by Iteration}

Figure 1 shows the principle of iterative ghost generation. An initial ghost, usually corresponding to zero-sum projections along the row or column direction, is chosen. This ghost has a trivial structure, requiring only 2 pixels, one with value +1 and the other with value -1 . The separation of these pixels is arbitrary, but to maximise ghost compactness, the two pixels are kept adjacent (Figure 1(a)). This pair of pixels is propagated into other discrete projection directions (for example along the column direction, Figure 1(b), and the diagonal direction, Figure 1(c)). A sign-reversed copy of the initial ghost is added at 


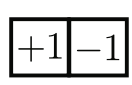

(a)

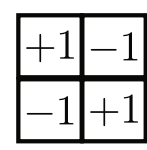

(b)

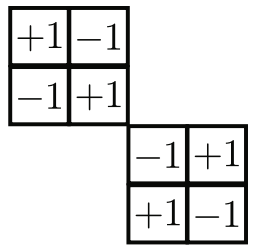

(c)

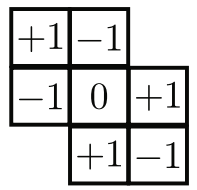

(d)

Fig. 1. Pairs of +1 and -1 pixels can be displaced and added iteratively to produce cancellation in new directions. A horizontal ghost a) is shifted vertically b) and then diagonally c) to produce a ghost that is invisible at three projection directions: 0,45 and 90 degrees. d) The third step can be translated so that one pixel with a +1 value has the same location as a pixel with a -1 value. This reduces the number of image pixels to $2^{N}-2$.

some displacement along a new angle, ensuring cancellation of the sums in both original and the new direction is preserved. This process is repeated to include all of the angles for which the ghost must sum to zero. After $N$ steps it is possible to attain a maximum image intensity of $2^{N}$ if there is no overlap of the +1 and -1 values at the same pixel location after the dilation.

The sign-reversed ghost can be deliberately translated to ensure the next -1 valued pixel is superimposed on the location of the last +1 valued pixel. This method always cancels at least two of the current ghost pixels and reduces the spatial extent and rate that pixel intensities will increase under subsequent iterations. The rate of growth of pixel intensities in iterated ghost images still remains $O\left(2^{N}\right)$.

\section{Ghost Generation by Row vs. Column Oriented Iteration}

The FRT, $R(t, m)$, projects data from a symmetric $I(x, y)$ space of size $p \times p$. However the row $(x)$ direction is arbitrarily chosen as the $t$ axis which represents the displacement of each projected ray. The projection angle $m$ accumulates as displacements along the column $(y)$ direction at step $\langle m y\rangle_{p}$. The FRT projections thus turn out to be sensitive to the row or column symmetry of any process we apply.

A simple algorithm is given in Algorithm 1 to describe iterative ghost construction, in an initially blank $p \times p 2 \mathrm{D}$ image space, $A(x, y)$.

Here the function $C S$ denotes a circular shift of the matrix $A$. The operation $C S$ moves the origin of the matrix $A$ to the point $(x, y)$. The pixel locations (and the pixel content) wrap modulus $p$ under circular shifts. The algorithm is iterated $N-1$ times to produce the set of $N$ required discrete projection angles. Gradient $x_{i}: y_{i}$ corresponds to an angle at $x_{i}$ steps across and $y_{i}$ steps down.

For row displacements, the circular shift parameters take the values $1 \leqslant x_{i} \leqslant$ $N$ and $y_{i}=1$. An example of the ghosts produced by this method is given in 


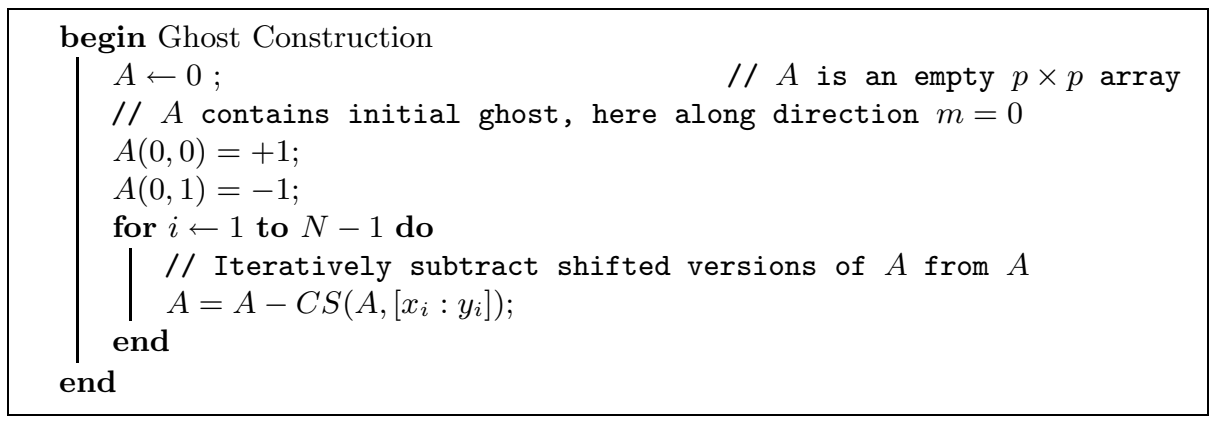

Algorithm 1. A simple algorithm describing iterative ghost construction

Figure 2(a) and the FRT of that ghost image is shown in Figure 2(b). Because the displacements are here $m$ steps across for each step down ( $m: 1$ gradients), the directions of the zero-sum ghosts correspond to sequential values of $m$. The sum of the absolute values of all pixels in row-dilated images is always exactly $2 \mathrm{~N}$ after $N$ row-oriented dilations. The spatial extent of the ghost after 8 dilations, as expected, covers 9 full image rows.

For column displacements, the circular shift parameters are $x_{i}=1$ and $1 \leqslant$ $y_{i} \leqslant N$. An example of the ghosts produced by this method is given in Figure 3(a) and the FRT of that ghost image is shown in Figure 3(b). Because the shifts here are one step across for $m$ steps down (1: $m$ gradients), the directions of the zero-sum ghosts are sequential values of $1 / m=m^{-1}(\bmod p)$. The angles are hence sequential in the inverse values of $m$, the integer angle parameter.

The sum of the absolute values of all pixels in column-displaced images after $N$ dilations varies slowly with $N$, as shown in Table1. The spatial extent of the ghost after 8 dilations covers 8 image rows (the initial ghost, $m=0$, is a column entry).

Table 1. Growth of the dynamic range of the absolute values of the ghost pixels after $N$ iterations along the row and column directions. The "sum absolute" figure is the integrated intensity of the absolute value of all the ghost image pixels for each $N$. The values here are computed for an array size $p=127$.

\begin{tabular}{lcccccc}
\hline Number of ghosts, $N$ & 7 & 8 & 10 & 12 & 16 & 20 \\
\hline Row Iteration & \multicolumn{7}{l}{} \\
\hline $\min / \max$ & $-5 / 5$ & $-6 / 8$ & $-20 / 18$ & $-49 / 58$ & $-468 / 526$ & $-4968 / 5448$ \\
\hline Sum absolute & $2^{7}$ & $2^{8}$ & $2^{10}$ & $2^{12}$ & $2^{16}$ & $2^{20}$ \\
\hline Column Iteration & & & & & & \\
\hline min/max & $-1 / 1$ & $-1 / 1$ & $-2 / 2$ & $-4 / 4$ & $-21 / 21$ & $-146 / 146$ \\
\hline Sum absolute & 26 & 40 & 104 & 304 & 3040 & 34224 \\
\hline
\end{tabular}



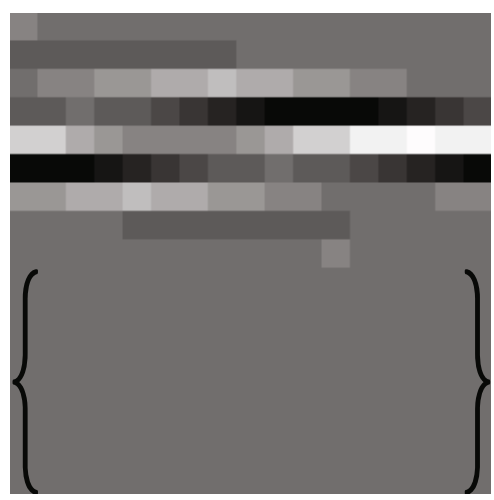

(a)

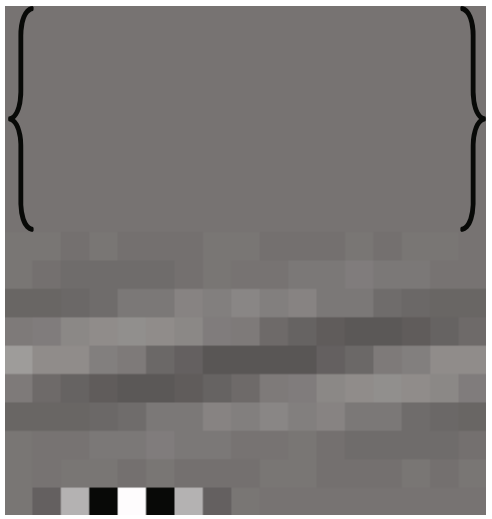

(b)

Fig. 2. (a) A $17 \times 17$ ghost image $I(x, y)$ with $N=8$ zero-sum projections created by row-oriented iterated convolutions. (b) the FRT, $R(t, m)$, of (a). The first 8 rows corresponding to $m=0$ to 7 are zero-sum projections. The pixel values in (a) range from -6 (shown as black) to +8 (shown as white). Mid-grey corresponds to a value of 0 . The summed pixel values in (b) range from -56 to +70 . The bracketed regions enclose empty image and projection space, respectively. The large values on the bottom row of (b) are the row sums $(m=p)$ of the pixel values shown in the image (a). These values accumulate rapidly for row-oriented displacements.

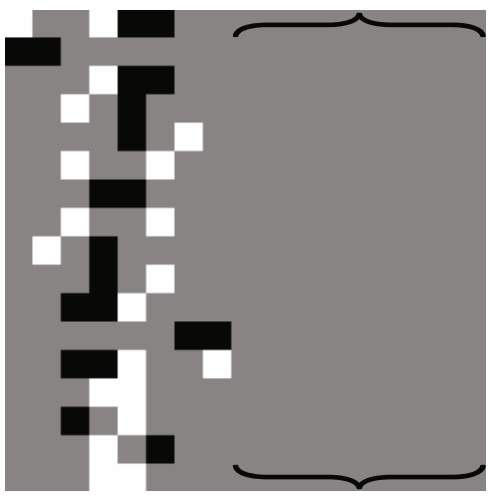

(a)

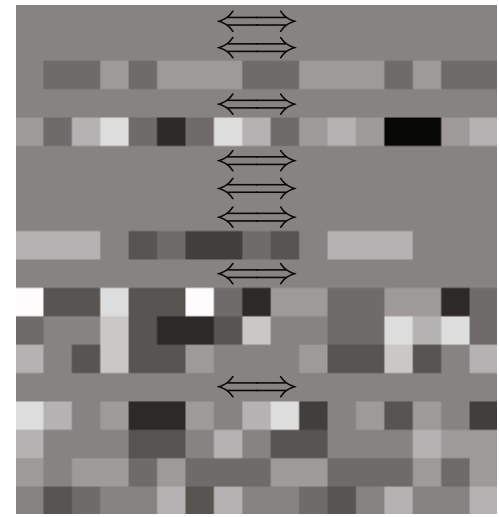

(b)

Fig. 3. (a) A $17 \times 17$ ghost image $I(x, y)$ with $N=8$ zero-sum projections created by column-oriented iterated convolutions. (b) The FRT, $R(t, m)$ of (a). The 8 rows corresponding to $m=\{0,1,3,5,6,7,9,13\}$ are zero-sum projections. The black arrows point to these zero-sum projections. The pixel values in (a) range from -1 (shown as black) to +1 (shown as white). Mid-grey corresponds to a value of 0 . The summed pixel values in (b) range between -6 to +8 . The bracketed portion of the image space in (a) is empty. 


\section{Growth of Iterated Ghosts}

Table 1 shows examples of the growth of ghost image pixel values under the two methods outlined above.

The values given in Table 1 are sensitive to $p$ when $N \approx p$ because of the strong overlap of pixel positions after each wrapping. For $p \gg N$, the ghost pattern wraps, at modulus $p$, around the array in a clean spiral pattern that causes minimal pixel position overlaps. In Table 1 $p=127$ and the maximum value of $N$ is 20 .

A log plot of the mean absolute ghost image pixel value $\left(g_{\max }\right)$ as a function of the number of ghost angles, $N$, is well fitted by a straight line. For the column displacements, $\log _{2}\left(g_{\max }\right) \approx 0.7(N-9)$. For the row displacements, $\log _{2}\left(g_{\max }\right) \approx$ $0.8(N-5)$. The value of $N$ at which we observe the onset of pixel intensities rise above +1 and -1 is smaller for the row-based dilations. The rate of growth of ghost image pixel values is significantly greater for the row-based approach rather than for column-based iteration.

Examples of ghost images at a larger $N$ and image scale are given in Figure 4 . Despite the large range of pixel values required to construct these ghosts, their spatial extent is quite small (being confined to 20 rows or columns respectively for $N=20$ ). The small spatial footprint of iterated ghost images may be a significant advantage in many applications [24].

Applying affine mappings (computed modulus $p$ ) to $N$ zero-sum projection ghost images such as those in Figure 5 spreads the ghost pixels entries across the image space. However the resultant affine-mapped ghost images still have $N$ zero-sum projections, albeit at a new set of FRT angles.

\section{Further Work and Conclusions}

The multi-valued ghosts shown here have been used in "de-ghosting" applications where rows of missing image data can be recovered exactly [4,2]. There we exploited the fact that the correct data is known to have zero or constant value across redundant parts of the image space. Missing data causes non-zero reconstructions in those regions that should be zero. Missing projection data can be regarded as being comprised of linear combinations of scaled and shifted ghost images.

The ghost images in Figure 4 are reminiscent of the Gabor oriented-filters used in texture analysis. It would be interesting to pursue this connection, as the 1D Fourier transform of each FRT row corresponds directly to a 1D slice in 2D FFT space.

The great advantage of ghosts constructed from only +1 and -1 pixel values is that they can be used to create a mixture of image and anti-image data where the combined image then has zero-sum projections at $N$ angles. This type of "expanded" ghost was used in [13] to encode redundancy into data for forward error correction in a proposed data transmission scheme. For small $N(N<9)$, 


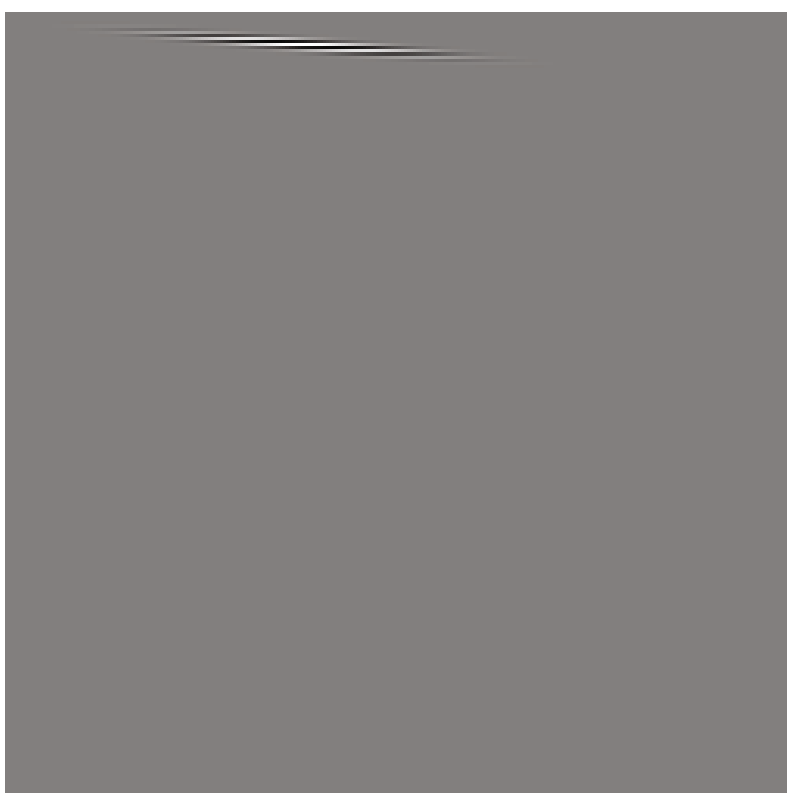

(a)

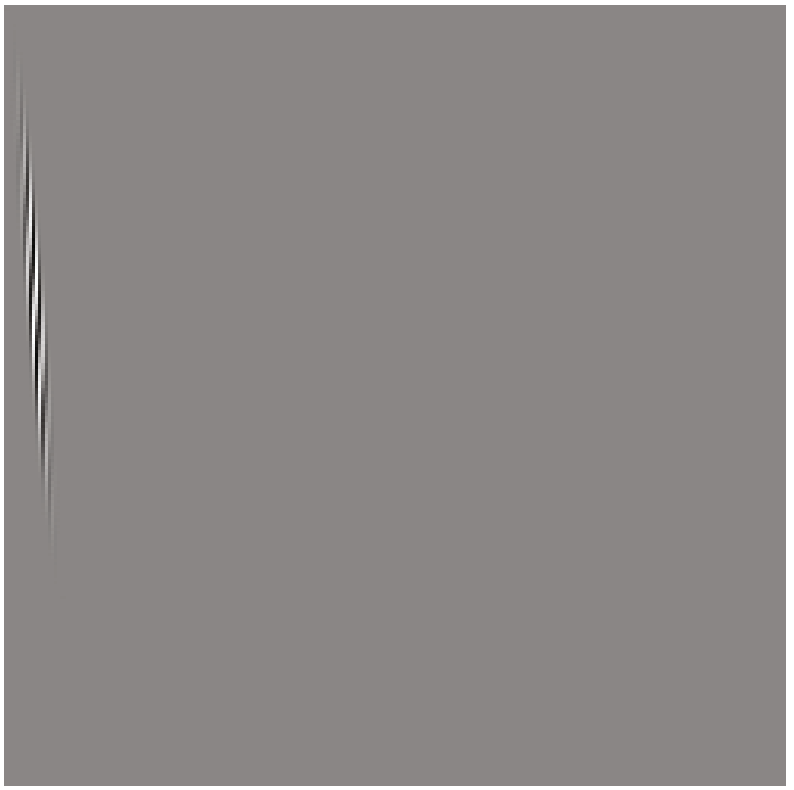

(b)

Fig. 4. Two $251 \times 251$ ghost images, each image has $N=20$ zero-sum projection angles. The row-iterated ghosts in (a) have pixel values that range from -4968 to +5448 . The values in (b), for column-iterated ghosts, range from -146 to +146 . The original image intensities have been both normalised to the range $0-255$ for display. 


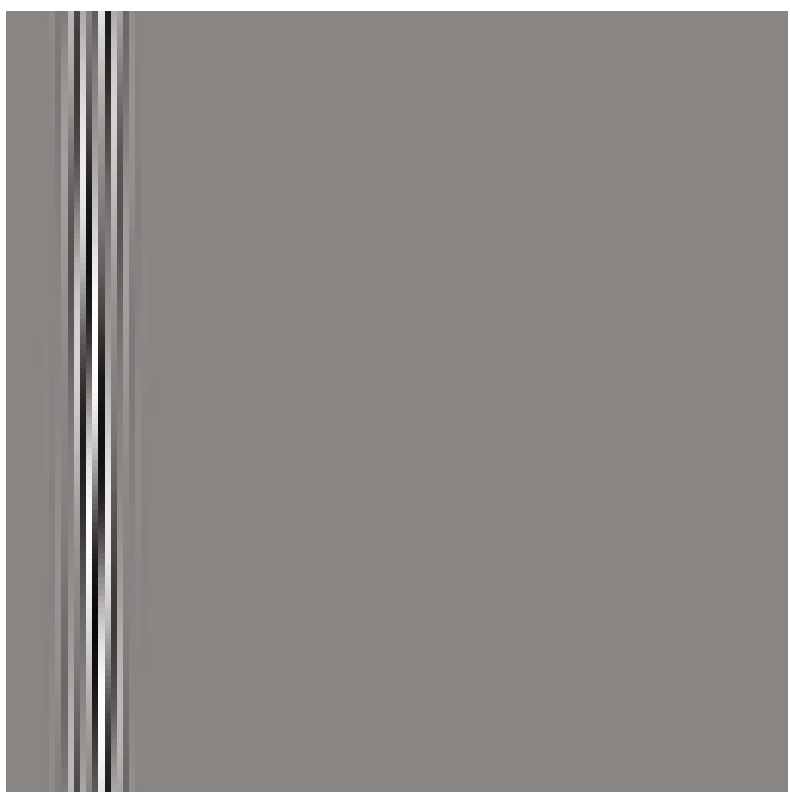

(a)

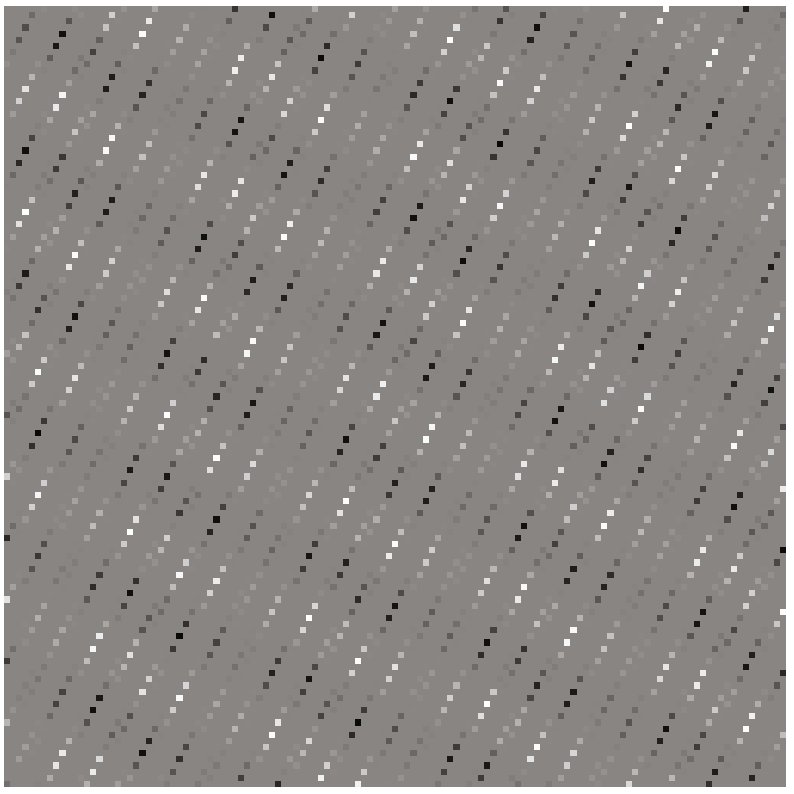

(b)

Fig. 5. A $127 \times 127$ ghost image with $N=29$ zero-sum projections. Here the columniterated ghosts have wrapped around the array from bottom to top. (b) Affine transform of (a) is still an $N=29$ ghost image, but at a new set of FRT angles. The same pixel intensities are now mapped uniformly across the image space in (b). 
the dilation method yields ghosts with pixel values restricted to \pm 1 . For these cases it is possible to keep a single +1 pixel at $(0,0)$ and reorder the pixel placements on subsequent rows whilst preserving the ghost properties.

Adding a copy of a negated ghost to a +1 pixel position cancels that pixel value to zero. Applying this process repetitively enables creation of a large blank space within the ghost structure that makes it suitable for image/anti-image generation (see Figure 6). The increase in pixel values of the overlaid ghosts causes larger intensities in the anti-image as it is synthesized from scaled image values multiplied by the brightness of the lower components of the ghost data. The addition of displaced ghost images causes further lateral spread of the ghost pixels. This method of creating "clear space" in a ghost image is then limited to clearing rows until the ghost image/anti-image wraps around to the top of the array.

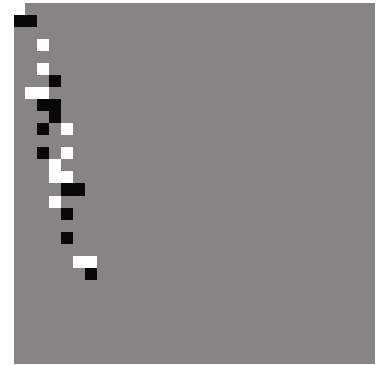

(a)

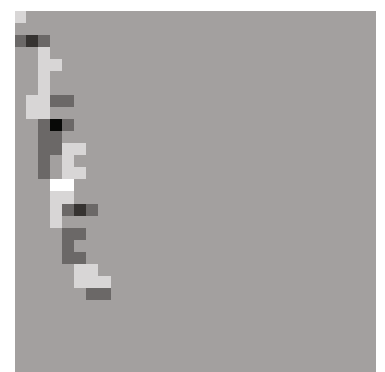

(b)

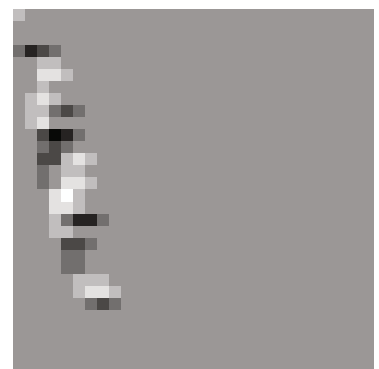

(c)

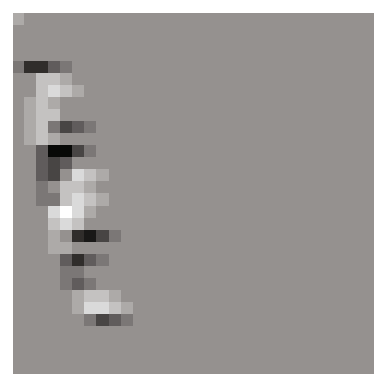

(d)

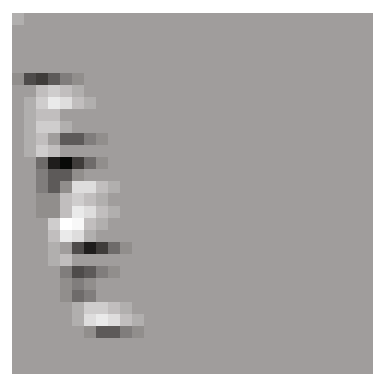

(e)

Fig. 6. The +1 pixel in the top left corner of the top row of the ghost image is retained in the sequence (a) through (e). Translated copies of image (a) are added to the second row of (a) to produce image (b), which has zero pixel values on row 2 . The process is repeated to zero the pixel entries on row 3 in (c), row 4 in (d) and row 5 in (e). The ghost image in (e), being composed of translated and scaled copies of the ghost image in (a) has exactly the same zero-sum projection rows after applying the FRT. The original image intensities in (a) through (e) have been normalised to the range $0-255$ for display. 
Acknowledgement. S.C. acknowledges the past support of a scholarship funded through the School of Physics and Faculty of Science at Monash University whilst completing his PhD. I.S. acknowledges ongoing support from the School of Physics at Monash for this work.

\section{References}

1. Barcucci, E., Del Lungo, A., Nivat, M., Pinzani, R.: X-rays characterising some classes of discrete sets. Linear Algebra and its Applications 339, 3-21 (2001)

2. Chandra, S.S., Normand, N., Kingston, A., Guédon, J.P., Svalbe, I.: Fast Mojette Transform for Discrete Tomography. Elsevier Signal Processing Submitted June (in Review), Available on arXiv.org (2010), http://arxiv.org/abs/1006.1965v1

3. Chandra, S.S., Svalbe, I.: A Fast Number Theoretic Finite Radon Transform. In: Proceedings of the Digital Image Computing Techniques and Applications Melbourne (December 2009), http://dx.doi.org/10.1109/DICTA.2009.67

4. Chandra, S., Svalbe, I.D., Guédon, J.-P.: An exact, non-iterative mojette inversion technique utilising ghosts. In: Coeurjolly, D., Sivignon, I., Tougne, L., Dupont, F. (eds.) DGCI 2008. LNCS, vol. 4992, pp. 401-412. Springer, Heidelberg (2008)

5. Gardner, R.J., Gritzman, P.: Discrete tomography: determination of finite sets by x-rays. Trans. Amer. Math. Soc. 349(6), 2271-2295 (1997)

6. Grigoryan, A.M.: New algorithms for calculating the discrete Fourier transforms. J. Vichislit. Matem. i Mat. Fiziki 25(9), 1407-1412 (1986)

7. Guédon, J.P., Normand, N., Kingston, A., Parrein, B., Servières, M., Évenou, P., Svalbe, I., Autrusseau, F., Hamon, T., Bizais, Y., Coeurjolly, D., Boulos, F., Grail, E.: The Mojette Transform: Theory and Applications. ISTE-Wiley, Chichester (2009)

8. Herman, G.T., Davidi, R.: Image reconstruction form a small number of projections. Inverse Problems 24, 17 (2008)

9. Katz, M.: Questions of Uniqueness and Resolution in Reconstruction from Projections. Lecture Notes in Biomathematics. Springer, Heidelberg (1977)

10. Kingston, A., Svalbe, I.: Projective transforms on periodic discrete image arrays. Advances in Imaging and Electron Physics 139, 75-177 (2006)

11. Louis, A.K.: Picture reconstruction from projections in restricted range. Mathematical Methods in the Applied Sciences 2, 209-220 (1980)

12. Matúš, F., Flusser, J.: Image Representation via a Finite Radon Transform. IEEE Transactions on Pattern Analysis and Machine Intelligence 15(10), 996-1006 (1993), http://dx.doi.org/10.1109/34.254058

13. Normand, N., Svalbe, I.D., Parrein, B., Kingston, A.M.: Erasure coding with the finite Radon transform. In: IEEE Wireless Communications \& Networking Conference, Sydney (April 2010), http://dx.doi.org/10.1109/WCNC.2010.5506385

14. Svalbe, I., Normand, N.: Properties of minimal ghosts. In: Accepted for presentation at DGCI Nancy, France, April 2011 (2010)

15. Svalbe, I., Normand, N., Nazareth, N., Chandra, S.: On constructing minimal ghosts. In: APRS Conference, DICTA 2010, pp. 1-3 (December 2010)

16. Svalbe, I.: Exact, scaled image rotation using the Finite Radon Transform. Pattern Recognition Letters (2010) (in press), http://dx.doi.org/10.1016/j.patrec. 2010.06 .015

17. Zopf, S.: Construction of switching components. In: Kuba, A., Nyul, L.G., Palagyi, K. (eds.) DGCI 2006. LNCS, vol. 4245, pp. 157-168. Springer, Heidelberg (2006) 